\title{
DSP Based Induction Motor Torque and Parameter Identification
}

\author{
J. Ke and B. Wu \\ Dept. of Electrical and Computer Engineering \\ Ryerson Polytechnic University \\ Toronto, Ontario, Canada M5B 2K3 \\ jkelee.ryerson.ca \\ bwuQee.ryerson.ca
}

\author{
R. Sotudeh \\ Dept. of Electrical and Computer Engineering \\ University of Teesside \\ Middlesbrough, Cleveland, UK TSI 3BA \\ R.Sotudeh@teesside.ac.uk
}

\begin{abstract}
A practical induction motor torque and parameter measurement scheme is proposed without using any mechanical sensors. The torque and parameter calculation is performed by a digital signal processor (DSP) based on measured motor terminal waveforms. Since the motor parameters are required for torque calculation, a novel scheme is developed for parameter identification. The motor parameters can be extracted from the measured motor current and motor voltage when the motor, fed by inverters, $\mathrm{AC}$ voltage controllers or other equipment, is started under no load conditions. The proposed torque measurement scheme has the features of wide bandwidth and no need for mechanical sensors. Simulation and experimental results on a $5 \mathrm{hp}$ induction machine driven under various operating conditions are also given for verification.
\end{abstract}

\section{Introduction}

Induction motors are often fed by voltage/current source inverters and $\mathrm{AC}$ voltage controllers, whose output waveforms are usually modulated and therefore contain voltage/current harmonics. These harmonics will produce harmonic torques which may excite mechanical resonances if the frequency of harmonic torque is close or equal to that of mechanical resonant modes which are associated with the moment of inertia of the motor and its load [1]. As a result, the motor shaft, bearing coupling, or rotor may be broken down. In this case, an accurate torque measurement tool is required to investigate the resonant phenomenon.

The traditional approach to measuring the shaft torque of an induction motor is by using a torque transducer which is mounted between the shafts of induction motor and its mechanical load. This torque measurement scheme has several drawbacks such as high cost, complex mechanical assembly and limited bandwidth (typically less than $300 \mathrm{~Hz}$, which means that higher order harmonic torque cannot be detected). Furthermore, the torque transducer may introduce an additional mechanicial resonance mode into the drive system, which may produce oscillating torque components superimposed on actual torque waveform during motor acceleration or deceleration.

The main objective of this paper is to developed a practical user friendly DSP based torque and parameter measurement system without using any mechanical sensors. The motor torque is evaluated based on measured instantaneous current and voltage. As a result, the problems associated with the use of shaft torque transducer are eliminated.

\section{Motor Torque Identification}

The induction motor dynamic model used in this paper is illustráted in Fig. 1, where $R_{s}$ and $R_{r}$ are stator and rotor winding resistances, $X_{m}$ and $X_{\sigma}$ are magnetizing and total leakage inductances, all in per unit and referred to the stator side. $\vec{\lambda}_{s}$ and $\vec{\lambda}_{r}$ are space vectors, representing stator and rotor flux linkages respectively. $\omega_{m}$ represents rotor mechanical speed and $\theta$ is the angle between the stator axis and rotor axis.

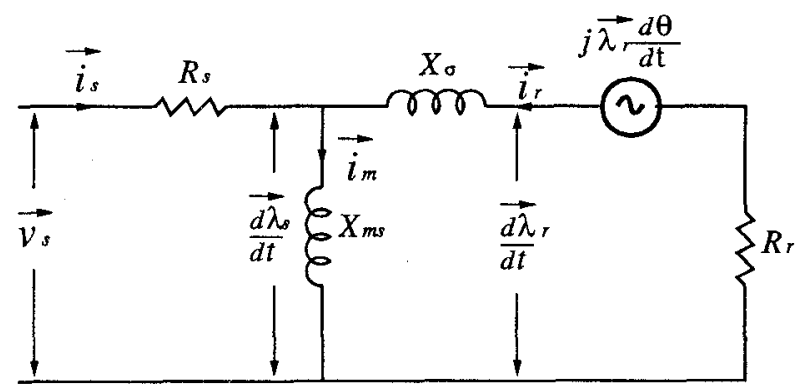

Figure 1: Dynamic model of induction motor in stator frame

The stator voltage can be expressed as

$$
\vec{v}_{s}=\vec{i}_{s} R_{s}+\frac{d \vec{\lambda}_{s}}{d t}
$$

where

$$
\vec{\lambda}_{s}=\left(\vec{i}_{s}+\vec{i}_{r}\right) X_{m s}
$$


The rotor flux linkage can be calculated by

$$
\begin{aligned}
\vec{\lambda}_{r} & =\vec{i}_{s} X_{m s}+\vec{i}_{r} X_{m s}+\vec{i}_{r} X_{\sigma} \\
& =\vec{i}_{m r} X_{m s}
\end{aligned}
$$

where $\vec{i}_{m r}$ is the equivalent rotor magnitizing current, which is a space vector as shown in Fig. 2. The relationship between $\vec{i}_{r}, \vec{i}_{m r}$ and $\vec{i}_{s}$ can be obtained by re-ranging Eq. 3, which is

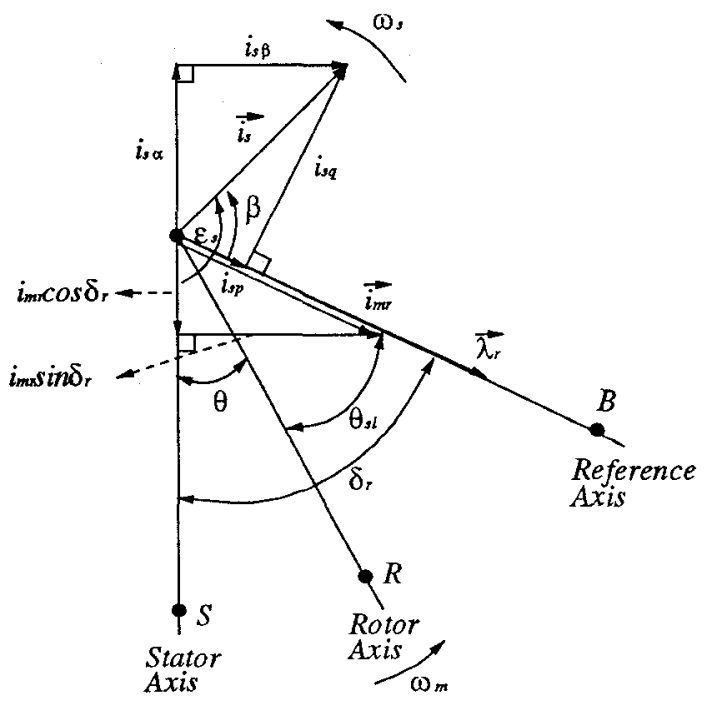

Figure 2: Induction motor space vector diagram

$$
\begin{aligned}
\overrightarrow{i_{r}} & =\frac{\vec{i}_{m r} X_{m s}-\vec{i}_{s} X_{m s}}{X_{m s}+X_{\sigma}} \\
& =\frac{\vec{i}_{m r}-\vec{i}_{s}}{1+\sigma}
\end{aligned}
$$

where $\sigma=\frac{X_{\sigma}}{X_{m}}$. Based on the stator flux linkage and stator current, the internal torque can be evaluated by

$$
\begin{aligned}
T_{q} & =\vec{\lambda}_{s} \times \vec{i}_{s} \\
& =\left(\vec{i}_{s}+\vec{i}_{r}\right) X_{m s} \times \vec{i}_{s} \\
& =\frac{\vec{i}_{m r}-\vec{i}_{s}}{1+\sigma} X_{m s} \times \vec{i}_{s} \\
& =\frac{X_{m s}}{1+\sigma} i_{m r} i_{s} \sin \beta \\
& =\frac{X_{m s}}{1+\sigma} i_{m r} i_{s q}
\end{aligned}
$$

where $i_{m r}$ and $i_{s q}$ are the magnitude of the equivalent rotor magnetizing current and the torque producing component of the stator current as shown in Fig. 2, respectively. To facilitate the torque evaluation, the equivalent rotor magnetizing current $\vec{i}_{m r}$ should be manipulated in such a way that it contains only the stator side variables. $\vec{i}_{m r}$ in Eq. 3 can be rewritten as

$$
\vec{i}_{m \tau}=\frac{\vec{\lambda}_{s}+\vec{i}_{\tau} X_{\sigma}}{X_{m s}}
$$

Substituting Eq. 4 into Eq. 6 yields

$$
\vec{i}_{m r}=\frac{(1+\sigma)}{X_{m s}} \vec{\lambda}_{s}-\sigma \vec{i}_{s}
$$

The stator flux linkage in Eq. 7 can be easily obtained by

$$
\vec{\lambda}_{s}=\int\left(\vec{v}_{s}-R_{s} \vec{i}_{s}\right) d t
$$

which can be decomposed into two scalar components.

$$
\lambda_{s \alpha}=\int\left(v_{s \alpha}-R_{s} i_{s \alpha}\right) d t
$$

and

$$
\lambda_{s \beta}=\int\left(v_{s \beta}-R_{s} i_{s \beta}\right) d t
$$

Similarly, Eq. 7 can also be separated into two equations (refer to Fig. 2 for details).

$$
i_{m r} \cos \delta_{r}=\frac{(1+\sigma)}{X_{m s}} \lambda_{s \alpha}-\sigma i_{s \alpha}
$$

and

$$
i_{m r} \sin \delta_{r}=\frac{(1+\sigma)}{X_{m s}} \lambda_{s \beta}-\sigma i_{s \beta}
$$

From Eqs. 11 and 12, the following equations can be derived.

$$
\begin{gathered}
i_{m r}=\sqrt{A+B} \\
A=\left[\frac{(1+\sigma)}{X_{m s}} \lambda_{s \alpha}-\sigma i_{s \alpha}\right]^{2} \\
B=\left[\frac{(1+\sigma)}{X_{m s}} \lambda_{s \beta}-\sigma i_{s \beta}\right]^{2} \\
\cos \delta_{r}=\left[\frac{(1+\sigma)}{X_{m s}} \lambda_{s \alpha}-\sigma i_{s \alpha}\right] / i_{m r}
\end{gathered}
$$

and

$$
\sin \delta_{r}=\left[\frac{(1+\sigma)}{X_{m s}} \lambda_{s \beta}-\sigma i_{s \beta}\right] / i_{m r}
$$

The torque producing component of the stator current in Eq. 5 can be calculated by

$$
i_{s q}=-\sin \delta_{r} i_{s \alpha}+\cos \delta_{\tau} i_{s \beta}
$$

Finally, the transformation between $a b c$ and $\alpha \beta$ quantities is given by

$$
\left[\begin{array}{c}
i_{s \alpha} \\
i_{s \beta}
\end{array}\right]=\left[\begin{array}{ccc}
1 & 0 & 0 \\
0 & -\frac{1}{\sqrt{3}} & \frac{1}{\sqrt{3}}
\end{array}\right]\left[\begin{array}{c}
i_{s a} \\
i_{s b} \\
i_{s c}
\end{array}\right]
$$

The same transformation is held for the stator voltages. Based on Eqs. 5, 9, 10, 13 . . 19, a block diagram for the torque evaluation is derived and given in Fig. 3. It is obvious that only stator voltages and stator currents need to be measured for the torque calculation. The speed signal is not required, which is one of the features of the proposed scheme. 


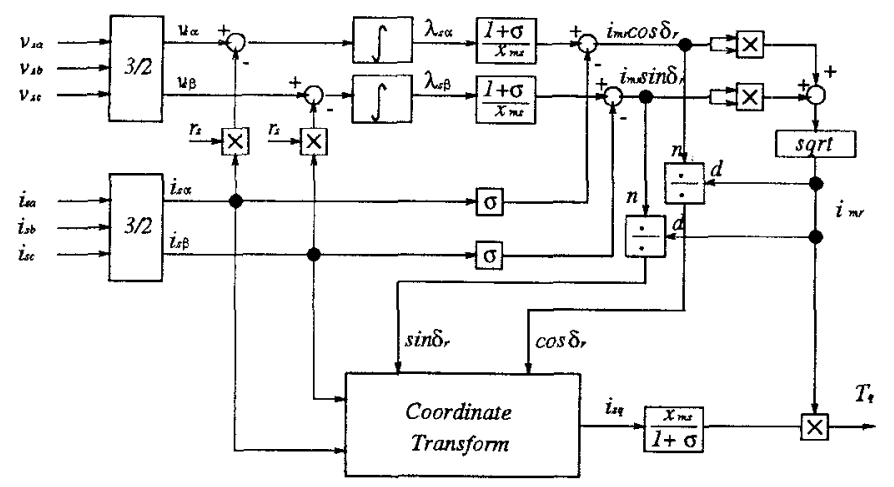

Figure 3: Block diagram of torque identification

\section{Motor Parameter Identification}

The motor torque measurement scheme discussed in the previous section requires motor parameters, such as stator and rotor winding resistances, total leakage inductance and magnitizing inductance. If these parameters are: available for a motor under test, the user can simply key in the parameters from the keyboard. However, the motor parameters are usually not available to the user. Therefore, an automatic parameter identification is necessary prior to the torque measurement. In what follows, an algorithm proposed in this paper for motor parameter identification is discussed.

The motor parameter can be identified when the motor, driven by an inverters or other power electronic equipment, is started from standstill to a certain speed under no load condition. It is assumed that during a very first cycles when the stator winding is energized, the rotor remains standstill due to its moment of inertia. This is especially true for large size motors. This assumption can also be achieved by reducing the voltage to frequency ratic applied to the motor under test. Since the starting torque is approximately proportional to the square of the stator voltage, reducing the voltage to frequency ratio will substantially increase the duration of the rotor acceleration process, which could be easily achieved by the inverter.

When the motor is started, the stator voltages and currents during the first few cycles are sampled and stored in the memory. Since the rotor remains at standstill, the motor dynamic model shown in Fig. 1 can be simplified. The simplified motor model is in fact the same as the one for the conventional blocked rotor test. Therefore the same calculation procedure applies for the calculation of motor parameters. When the motor reaches steady state under no load conditions, the stator voltages and currents are sampled for a couple of cycles. This is equivalent to the conventional no load test, based on which all the motor parameters can be identified.

It should be pointed out that the stator voltages and currents are rich in harmonics. A Low Pass (LP) filter should be used to eliminate the harmonics in the sampled signal prior to the motor parameter identification. A sinusoidal signal is expected at the output of the filter under steady state conditions. However, the filter may experience a transient when it is started with a zero initial condition. How to minimize the effect of the filter transient on the accuracy of motor parameter identification will be discussed in the next section.

\section{Implementation}

The motor torque and parameter measurement are implemented by using a low cost Motorola 56002 DSP evaluation board. An interface board is built which includes four A/D converters, two D/A converters and keypad. The sampling frequency of ADCs is $100 \mathrm{kHz}$. Due to a fixed point operation of 56002 digital signal processor (DSP), proper scaling and de-scaling are also considered. Fig. 4 shows the block diagram of hardware configuration. When a software controlled switch is connected to

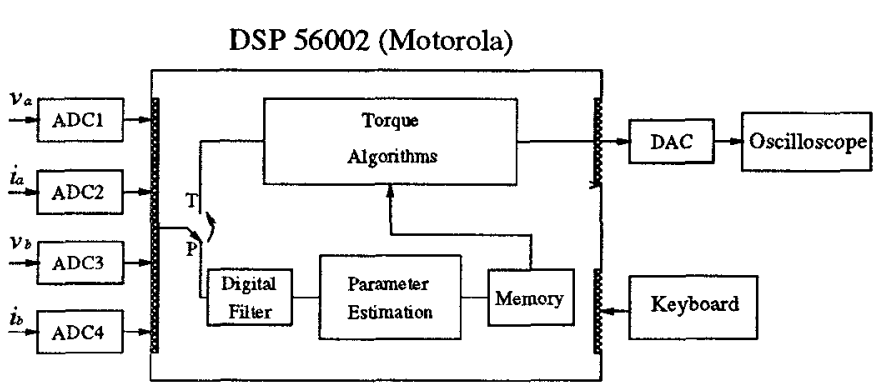

Figure 4: Block diagram of motor torque and parameter implementation

$P$, the DSP board performs motor parameter estimation and stores the estimated motor parameters in the memory. Then, the switch is switched to $T$, the motor torque calculation will be processed and the measured torque waveform is displayed on the oscilloscope via DAC.

\subsection{Integrator}

As shown in Fig. 3, digital filter are required for the torque identification. In practice, however, a pure integrator is not suitable because it is not capable of removing DC components during the torque calculation which otherwise will cause the integrator to be saturated. The undesired DC bias might be introduced to the system from either input signal or external circuitry. Therefore, the integrator should be designed in such a way that it can reject the DC components to avoid saturation. A practical integrator transfer function is given in Eq. 20 and its $\mathrm{S}$ domain plot is shown in Fig. 5. The corner frequency of modified integrator is around $1 \mathrm{~Hz}$, and thus the unwanted DC bias can be substantially attenuated.

$$
H(\dot{\mathbf{s}})=\frac{\dot{\mathbf{s}}}{\dot{\mathbf{s}}^{2}+1.047 \dot{\mathbf{s}}+1}
$$



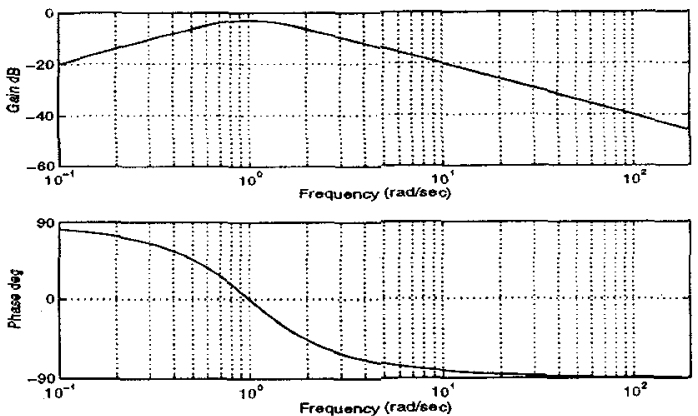

Figure 5: Bode plot of the integrator transfer function

\subsection{Low Pass Filter}

As mentioned in the previous section, the transient of LP filter should be minimized in order to increase the accuracy of the parameter identification. This is especially important when the motor is started from standstill during which only a first few cycles of the stator voltages and currents can be used for parameter calculation. This transient can minimized by selecting the right starting time of the filter. Fig. 6 and 7 illustrate the input and output signals of the LP filter. In Fig. 6, the filter with zero initial conditions is started at a zero crossing of input waveform, where the transient approximately lasts for one and half cycles. When the filter starts to operate at the peak of the input as shown in Fig. 7, the transient at the output is minimized. Therefore, in the DSP program, the LP filter is always started at the peak of the input or at $\omega_{t}=\frac{\pi}{2}$ or $\frac{3 \pi}{2}$.
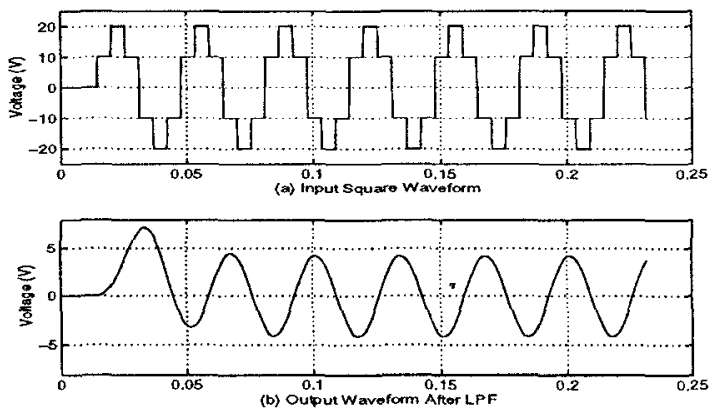

Figure 6: Input and output waveforms when the LP filter is applied at zero crossing of input signal

Waveform (a) in Fig 7 illustrates the input signal and waveform (b) shows the signal waveform at the output of the filter when filter is applied at the peak input signal.

\section{Simulation and Experiment Results}

The motor used in the simulation is rated at $208 \mathrm{~V}, 14.3 \mathrm{~A}$ and $5 \mathrm{hp}$. The parameters identified by using the algorithm proposed in this paper are listed in Table 1. This test was done when the motor was started by an IGBT based inverter at the frequency of $60 \mathrm{~Hz}$ and half rated voltage. The parameters measured by the conventional motor tests (no load and blocked rotor tests) are also listed in the table for comparison. It is obvious the test
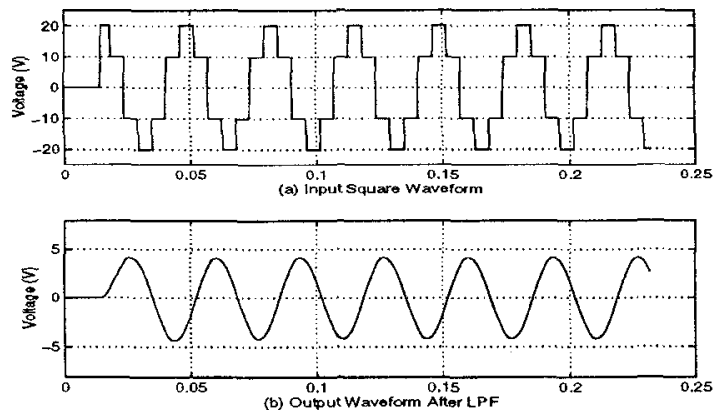

Figure 7: Input and output waveforms when the LP filter is applied at the peak of input signal

\begin{tabular}{|c|c||c|c|}
\hline $\begin{array}{c}\text { Motor } \\
\text { Parameters }\end{array}$ & $\begin{array}{c}\text { Conventional } \\
\text { Tests } \\
\text { Value }(\Omega)\end{array}$ & $\begin{array}{c}\text { Identified } \\
\text { Value }(\Omega)\end{array}$ & $\begin{array}{c}\text { Average } \\
\text { Error }\end{array}$ \\
\hline$R_{s}+R_{r}$ & 1.056 & $\mathbf{1 . 0 6 3}$ & $\mathbf{0 . 6 6 \%}$ \\
\hline$X_{\delta}$ & 1.603 & $\mathbf{1 . 6 1 7}$ & $0.87 \%$ \\
\hline$R_{s}+R_{m}$ & 1.815 & $\mathbf{1 . 8 7 1}$ & $3.09 \%$ \\
\hline$X_{m}$ & 18.883 & $\mathbf{1 8 . 2 4 4}$ & $-3.38 \%$ \\
\hline
\end{tabular}

Table 1: Identified motor parameters using the algorithm proposed in this paper

results by both methods are quite close. Fig. 8 and 9 illustrate simulated and measured stator and torque waveforms during a soft start-up of the $5 \mathrm{hp}$ induction motor.
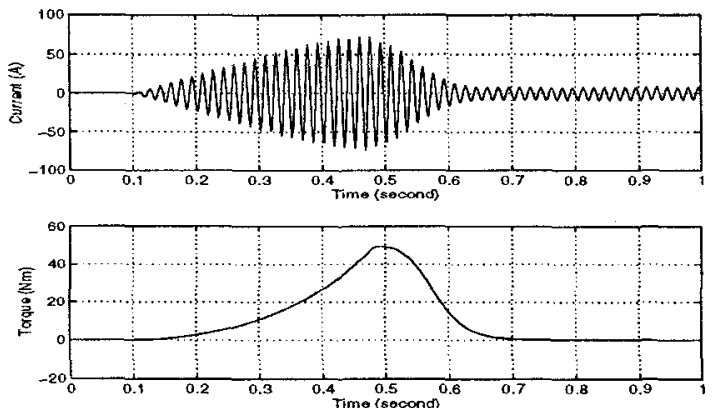

Figure 8: Simulated waveforms during motor soft start-up
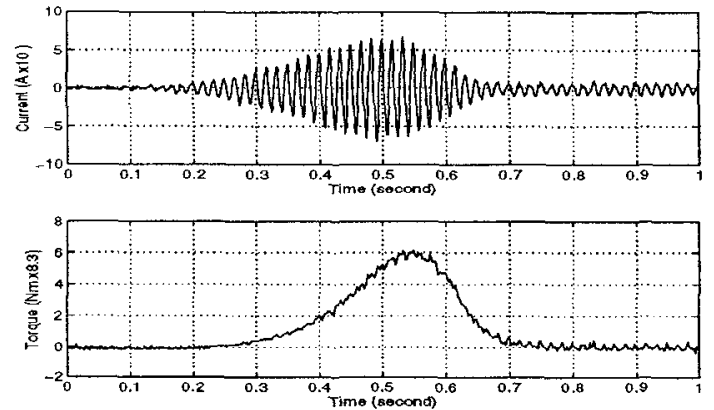

Figure 9: Measured waveforms during motor soft start-up

The stator voltage is a ramp function with time to avoid large inrush current. The simulated and identified torque waveforms are quite consistent. Figures 10 and 11 show the simulated and measured waveforms when the motor 
terminals connected to a $60 \mathrm{~Hz}$ supply are shorted during a fault.
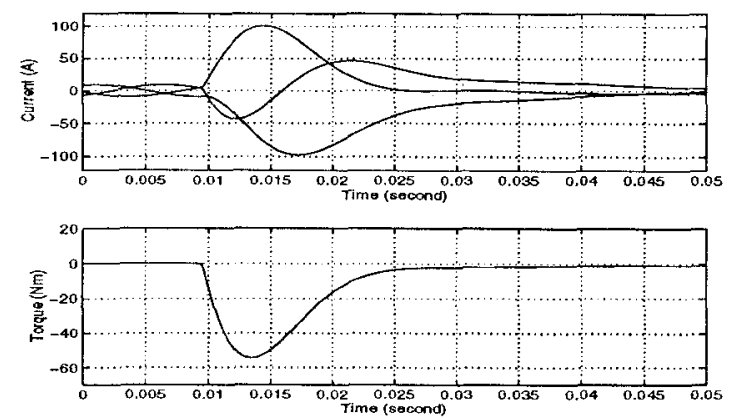

Figure 10: Simulated waveforms when the motor terminals are shorted during a power fault
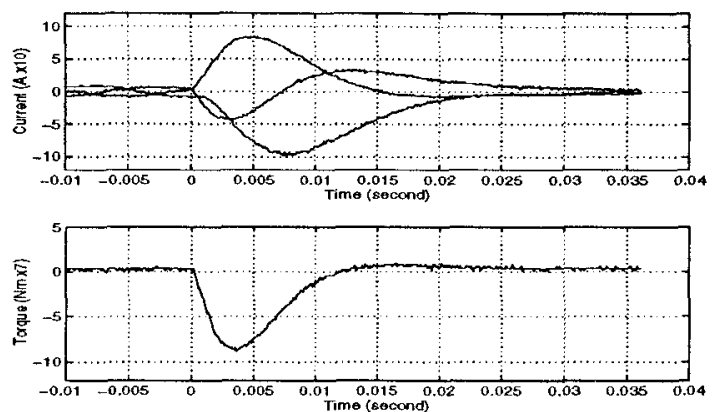

Figure 11: Measured waveforms when the motor terminals are shorted during a power fault
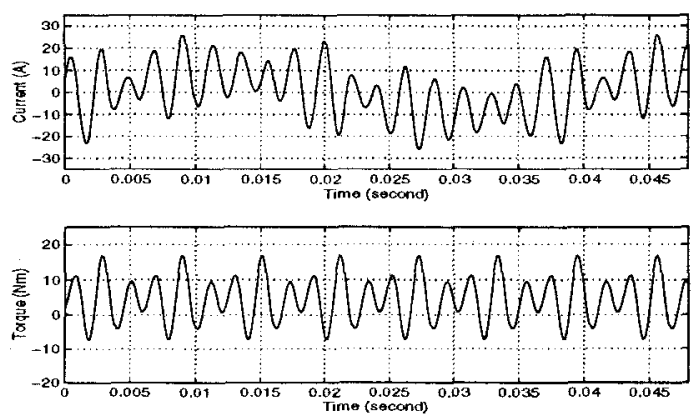

Figure 12: Simulated waveforms when the motor is fed by a PWM current source inverter. The PWM switching patterns are designed in such a way that resonances are excited on purpose
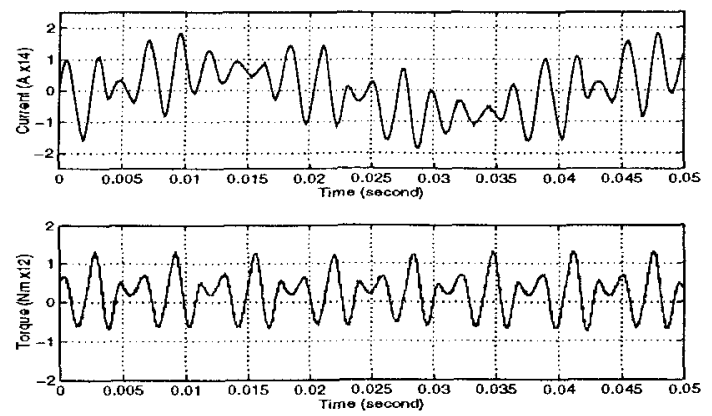

Figure 13: Measured waveforms under the same conditions as those given in Fig. 12

Figures 12 and 13 are the simulated and measured steady state waveforms when the motor is fed by a PWM current source converter. The PWM switching patterns are designed in such a way that the resonances between between the motor leakage inductance and inverter output capacitor are excited on purpose. The steady state torque waveform contains large 6 th and 12 th harmonic components. The simulated and measured waveforms shown in the above figures are well agreed.

\section{Conclusions}

A practical DSP based induction motor torque and parameter measurement scheme is proposed without using any mechanical sensors. The torque and parameter calculation is performed by a digital signal processor based on measured motor terminal waveforms and a well established dynamic model. Since motor parameters are required for the torque calculation, a practical scheme is developed for motor parameter identification. The motor parameters can be extracted from the measured motor terminal voltage and current when motor, fed by inverters, AC voltage controllers, or other equipment, is started under no load conditions. A few practical issues such as digital integrator design to avoid DC saturation are also investigated. The proposed torque measurement scheme has the features of automatic motor parameter identification, wide bandwidth and no need for mechanical sensors. Simulation and experimental results on a $5 \mathrm{hp}$ induction motor under various operating conditions are also given for verification.

\section{References}

[1] David J. Sheppard, "Torsional Vibration Resulting from Adjustable-Frequency AC Drives" IEEE Trans. on Ind. Aprl., Vol.24.No.5, Sep/Oct 1987, Pp812-817.

[2] Werner Leonhard, "Control of the Electrical Drives," Springer, New York 1996

[3] Tsugutoshi Ohtani, "A new method of torque control free from motor parameter variation in induction motor drives," IEEE Press, IAS-86, Pp203-209, 1986. 